\title{
Induced Axial Oscillations in Superconducting Dipole Windings
}

\author{
W.B. Sampson and A.K. Ghosh \\ Brookhaven National Laboratory, Upton, N.Y. 11973 U.S.A.
}

\begin{abstract}
When superconducting accelerator magnets wound from multi-stranded conductor are energized a periodic variation appears in the magnetic field along the axis. This oscillation is present in all components of the field and has a period that is equal to the transposition pitch of the superconducting cable. Such axial variations have been observed even in windings which are not carrying any transport current. A magnetic field was applied to a portion of a dipole winding using a second magnet. Axial oscillations were induced along the total length of the vindings including the portion not in the applied field. The amplitude of these oscillations varied with the amount of inert winding inside the energizing magnet and with the angle of the applied field. These field variations could be completely eliminated in the external portion of the coil by heating a small section of the winding above the transition temperature.
\end{abstract}

\section{INTRODUCTION}

Periodic variations in the sextupole field along the axis of superconducting dipoles were first observed at DESY in 1990 [1]. It soon became apparent that such field oscillations were characteristic of all superconducting accelerator magnets [24]. These axial variations are present in all the harmonic components of the field both skew and normal and have a wavelength equal to the transposition length of the superconducting cable. A typical example is shown in Fig. 1 for the dipole and normal sextupole components of a RHIC prototype dipole.

The mechanism which produces these oscillations is poorly understood, but they appear to be due to unbalanced currents in the individual strands of the cable. For all the magnets studied so far the field has been produced by the transport current in the coils themselves so that both field and current are changing simultaneously. In order to investigate this unusual phenomenon in detail an experimental arrangement in which the field and current were independent was assembled from apparatus available at BNL. A pair of prototype SSC coils were mounted in the bore of a test dipole and the field patterns measured to see if axial oscillations could be induced in windings which were not carrying any transport current.

\section{EXPERIMENTAL ARRANGEMENT}

Figure 2 shows the position of the unpowered coils in the high field dipole. The coils are mounted on a "warm finger" or anti-cryostat so that the measuring probes are at room

\footnotetext{
* Manuscript received October 18, 1994.

Work supported by the U.S. Department of Energy under Contract

Number DCO2-76CH00016.
}

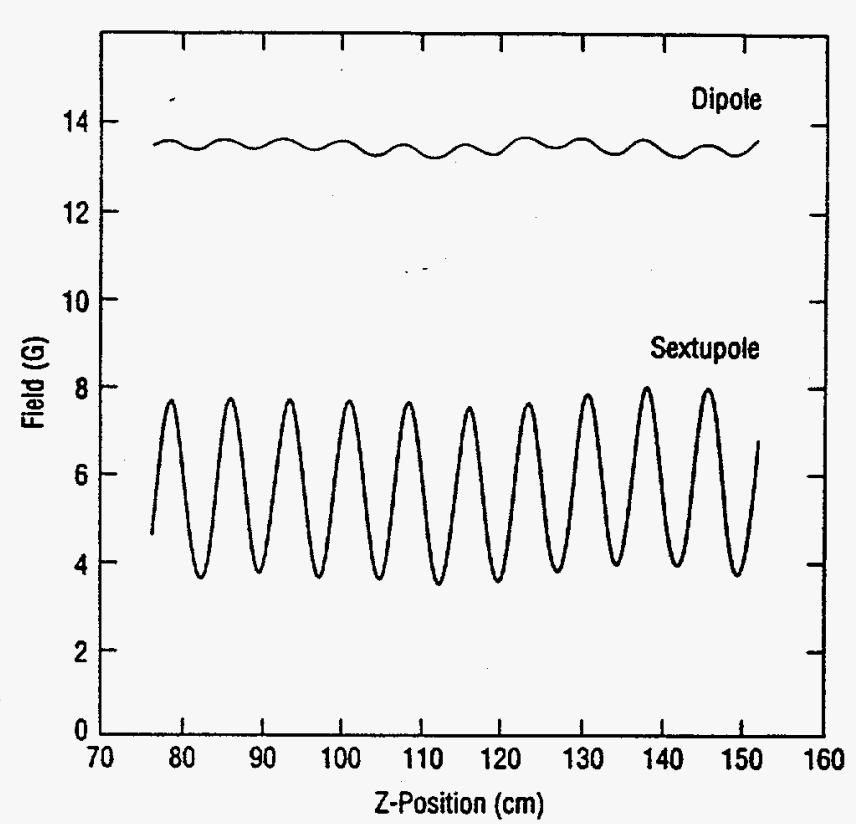

Figure 1. Axial oscillations in a section of a prototype RHIC $10 \mathrm{~m}$ dipole. The magnet has been held at $6000 \mathrm{~A}$ for $1 \mathrm{hr}$. and then measured at zero current.

temperature. SSC $4 \mathrm{~cm}$ short inner coils were used since they were available and are small enough to fit in the test magnet. Because of the difference in length approximately one half of the windings are in the field. The soldered ends of the SSC coils were removed and there was no connection between the two halves. Initially the poles were aligned with the dipole as shown in Fig. 3 but the complete assembly could be rotated and moved axially over a limited range. A stainless steel foil heater was installed on the windings near the end not in the field. This heater covered the complete circumference and could directly heat the edge of every turn in the unpowered winding over a length of $2 \mathrm{~cm}$.

The dipole used to produce the applied field is capable of a maximum field of $7.5 \mathrm{~T}$ and has a persistent switch for $\mathrm{dc}$ operation which limits the ramp rate to approximately 50 gauss/sec. This magnet is used on a daily basis to measure the electrical properties of superconducting cables and the experiments described in this paper were done by substituting the assembly mentioned above for the usual high current sample holder. Earlier measurements had shown that the axial field pattern produced by this magnet was very small compared to the SSC prototypes which had been tested. The reason for the unusually small oscillations in this magnet are not understood but may be related to the fact that the cable in this magnet was made from a continuous piece of conductor

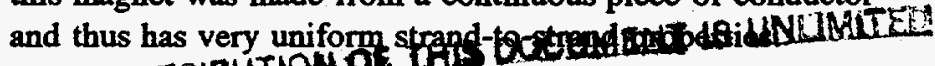




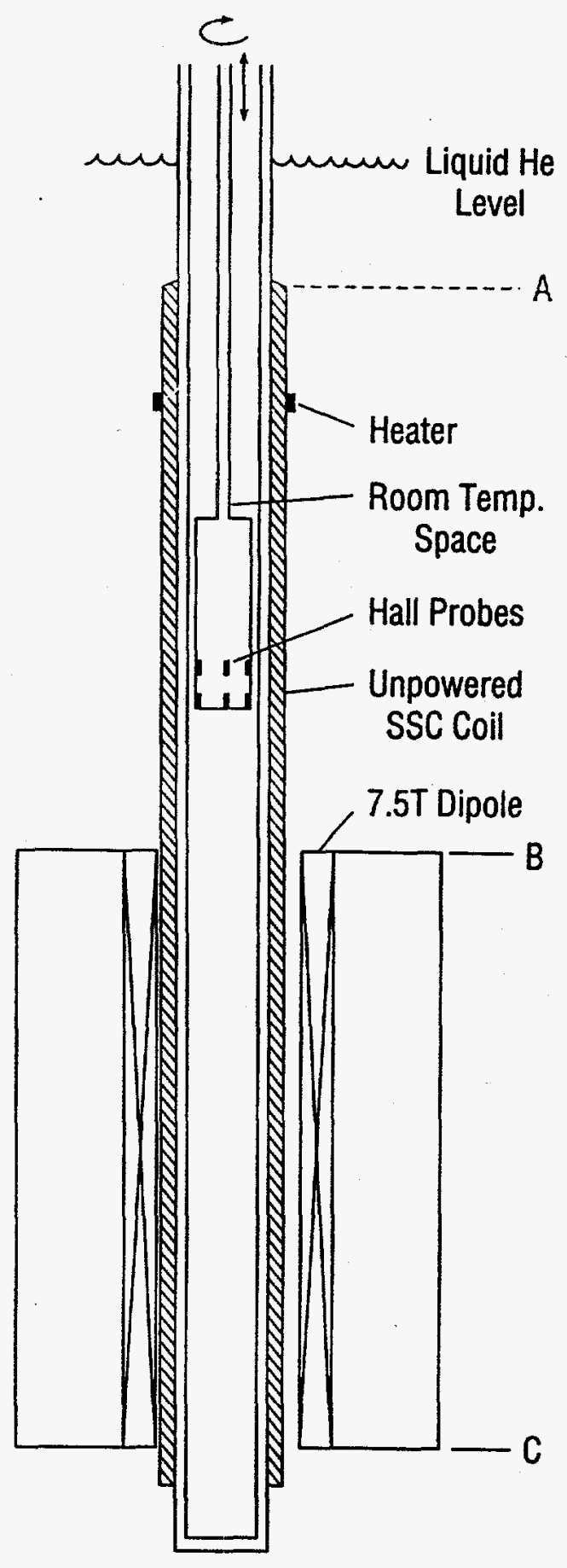

Figure 2. A schematic diagram of the experimental arrangement. The Hall probe array can be moved vertically and rotated automatically.

The measuring system used in this study is illustrated in Fig. 4 and consists of an array of eight Hall effect sensors in a solid aluminum form. This probe is connected by a long shaft to a transport mechanism which provides axial and rotational motion under the control of a small computer. The Hall sensors were chosen from a large batch ( $~ 40$ units) by

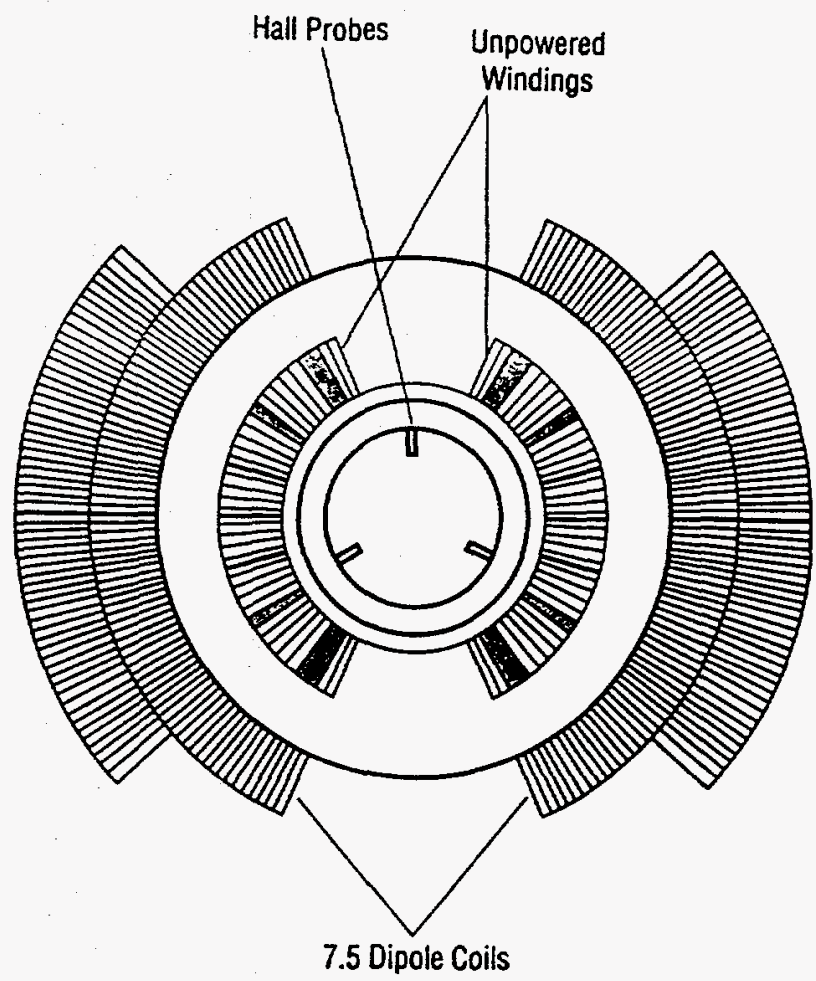

Figure 3. A cross section showing the relative position of the unpowered windings and the main dipole coils. The unpowered coils can be rotaled up to $60^{\circ}$ relative to this initial position.

direct measurement in a special magnet designed for this purpose. After all the sensors had been measured individually similar units were tested simultaneously by directly subtracting the output voltages. In this way two groups of four elements each with closely matched properties were selected. One pair (labeled 1 and 2 in Fig. 4) can be used to measure all field harmonics by rotating the probe holder through 360 degrees and analyzing the difference signal. Adding the outputs of the elements labeled 2,3 and 4 gives a signal directly proportional to the sextupole field without requiring rotation and can be used to scan the axis for this harmonic. A second set of four elements is positioned 4 $\mathrm{cm}$ from first group. Three heaters are evenly spaced around the probe form and extend over its length. A platinum resistance thermometer is imbedded in the aluminum form and used to control the heaters and keep the assembly at a constant temperature as it scans up and down through the coils.

\section{EXPERIMENTAL RESULTS}

The most interesting result was observed as soon as the dipole was energized. An axial oscillation signal appeared in the bore of the unpowered coils even in the portion that was not in the applied field. The amplitude of this oscillation increased as the field increased and decayed when the field was maintained at a constant level. When the field was 


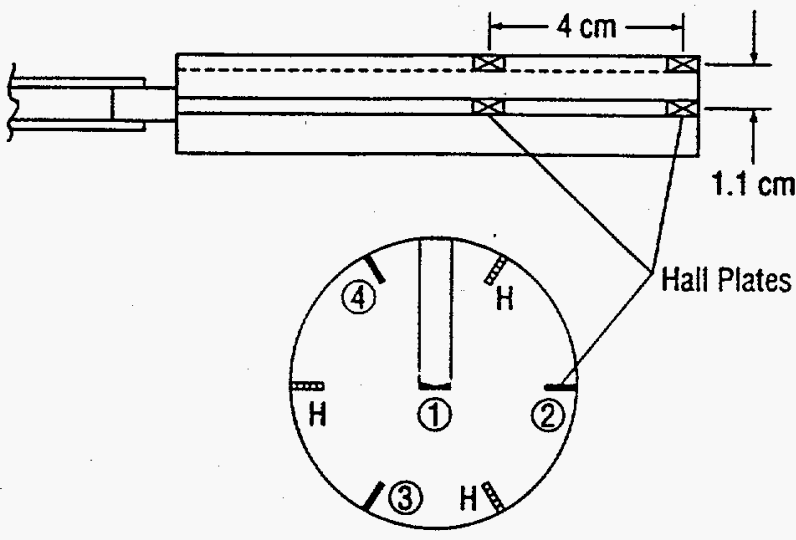

Figure 4. The position of the Hall sensors in the aluminum measuring probe. The henters extend over the total length of the probe and are indieated by the symbol $H$.

reduced the oscillation amplitude decreased, but due to the continuous decaying of the pattern an oscillation of the opposite sign is present when the dipole current is reduced to zero. The magnitude of this remnant pattern depends on how high the driving field is raised and how long it is maintained at this level since the amplitude decays more rapidly at higher levels. Figure 5 shows the remnant sextupole oscillation after a cycle up to $2 \mathrm{~T}$ and back to zero. The amplitude inside the dipole is twice as big as in the region outside the magnet. None of the signal observed inside the magnet is due to the

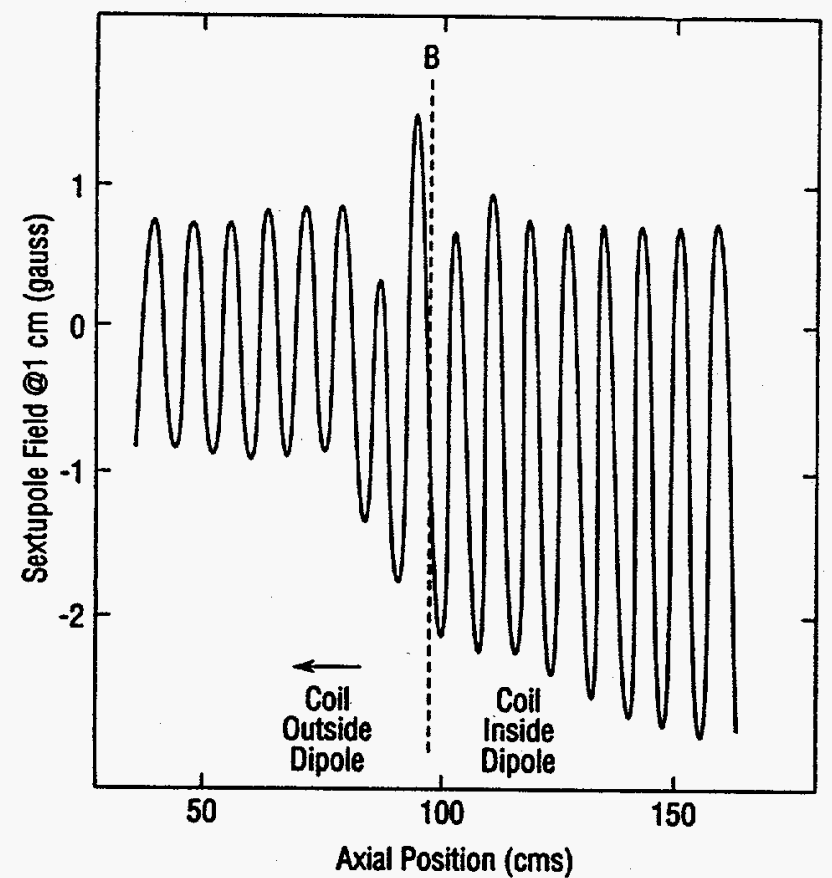

Figure 5. An axial scan through the unpowered winding after a field change of $4 \mathrm{Tesla}$. The dached line represents the end of the dipole magnet; point B in Fig. 2.

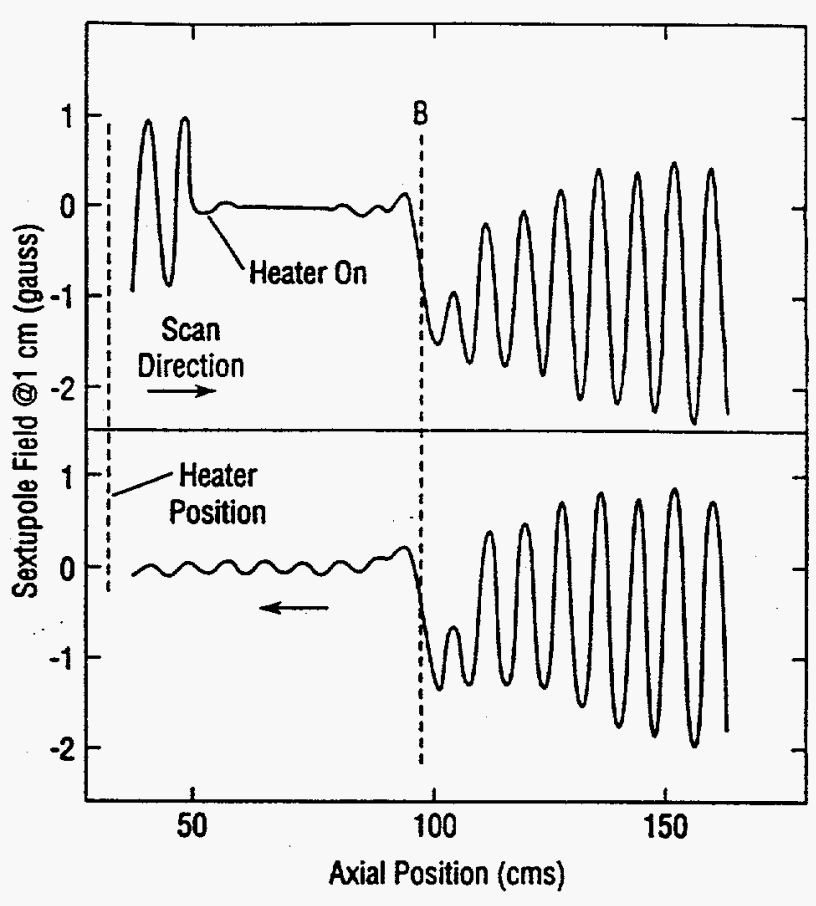

Figure 6. The effect of the coil heater being pulsed during an axial scan. The position of the henter is indicated by the broken line on the left in this figure.

dipole itself since the pattern turns with the unpowered winding when it is rotated. Once established the patterns lasts for a very long time, decaying rapidly at first and then very slowly. The amplitude of the axial pattern is measured by scanning a region covering several oscillations and using the same analysis method as in the rotating case. Typically three complete oscillations are included and the magnitude appears as the third harmonic in the Fourier analysis. This technique can be used to determine the exact wavelength by adjusting the scan length to maximize the appropriate harmonic. Oscillations occur in both the skew and normal sextupole terms but the values depicted in the figures are all for the normal component for simplicity.

When the heater is energized the axial pattern behaves in an unusual way. As the heater current is increased nothing changes until a critical value is reached when the pattern outside the magnet vanishes almost instantaneously. This is illustrated in the top of Fig. 6 where the heater is turned on during a scan. Inside the dipole the pattern is reduced but does not disappear. The bottom trace of Fig. 6 shows that a small oscillation reappears by the time the probe returns after passing through the dipole. A second heater pulse will eliminate this small oscillation which only reappears when the heater is on for a very short time. If the heater is used to eliminate the axial pattern at field an oscillation of opposite polarity appears when the magnet is turned down. Using the heater it is possible to start at any position on the magnet energizing curve and raise or lower the field to produce appropriate patterns. This can only be done for the region outside the magnet of course since all the signal does not 


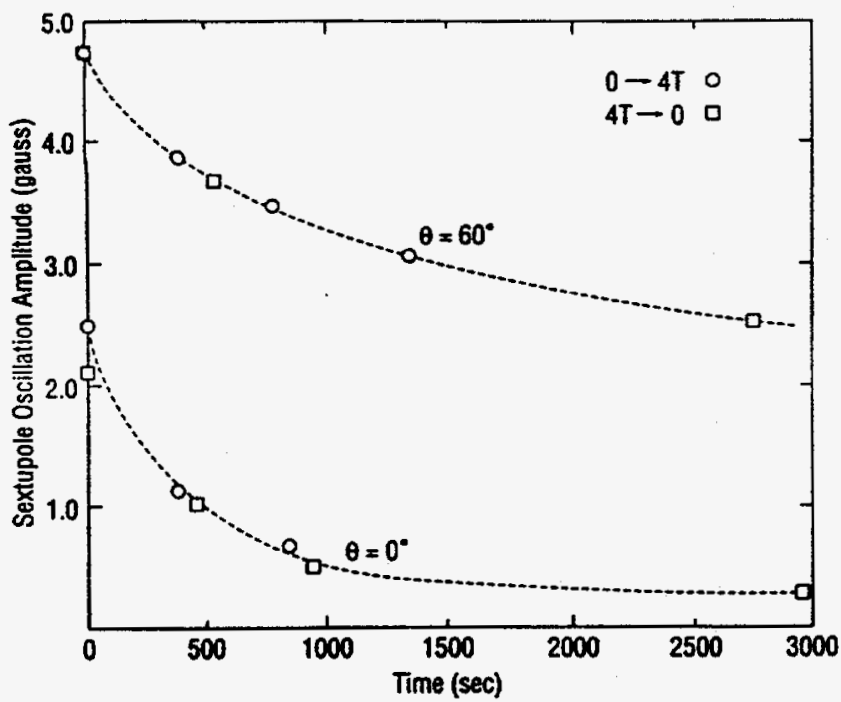

Figure 7. Time dependence of oxcillation amplitude induced by a 4T field change for two angular orientations of the unpowered winding. The initial position of Fig. 3 is represented by $\theta=0^{\circ}$.

damp out inside the magnet when the heater is pulsed.

Using this technique the amplitude induced by a step in field was investigated. The signal between $40 \mathrm{~cm}$ and $65 \mathrm{~cm}$ (three oscillations) was measured under the following conditions: First, the heater was used to eliminate any oscillation; then the field was increased to $1.3 \mathrm{~T}(500 \mathrm{~A})$ and the amplitude measured. After erasing the signal at this level the field was increased to $2.6 \mathrm{~T}$, an equal step in field, and the amplitude remeasured. Again the pattern was eliminated by heating and the field increased to $3.9 \mathrm{~T}$. This procedure was repeated for the same steps on the way down. For each of these field steps the oscillation amplitude was found to be the same ( 0.37 Gauss rms) within the accuracy of the measurement (.01 Gauss rms) and to have the same time dependence. This experiment was repeated using different ramp rates with the same result when the effect of decay during the slow ramps is taken into account. Due to the presence of the persistent mode switch it was not possible to achieve field change rates comparable to those of previous self-powered magnet tests [4].

The effect of moving the unpowered coil relative to the dipole was examined by measuring the amplitude of oscillation induced by a $4 \mathrm{~T}$ field change as the coil was raised in $1 \mathrm{~cm}$ steps. Initially the amplitude decreased to approximately $60 \%$ of the initial value but then increased by almost two and one half times when the assembly had been moved one wavelength $(8 \mathrm{~cm})$. Unfortunately it was not possible to systematically investigate this behavior over a longer distance because of interference with other equipment. At this position $(+8 \mathrm{~cm})$ the unpowered coil assembly was rotated relative to the dipole and subjected to the same $4 \mathrm{~T}$ field change. The oscillation amplitude increased by almost a factor of two at an angle of $60^{\circ}$. Again interference with other apparatus prevented a systematic study of the angular dependence.

\section{DISCUSSION AND CONCLUSIONS}

The fact that axial patterns appear in the part of the coil not in the applied field implies that unbalanced currents are induced in the strands of the conductor and that the phenomenon is not a magnetization effect. The heater experiments are consistent with this since the oscillations vanish at a discrete current and are not changed by thermal inputs only slightly less than this critical value. Since these circulating currents are a very small fraction of the critical current, the heater must raise the temperature of the conductor to its critical value $T_{c}$, to quench them. Because the pattern is approximately uniform over the whole external region and the beating of a very small section of this region eliminates the oscillations along its total length, a coherent distribution of currents that pass from one end of the winding to the other must exist. Inside the dipole the oscillation amplitude is larger suggesting that some of the induced currents are reflected by the discontinuity represented by the end of the high field region. The two sections of the coil behave somewhat like coupled resonant cavities. When the applied field is changed currents are induced in the conductor that reflect from the ends of the coil with a partial reflection at the dipole end. The heater quenches the currents which travel from end to end but does not affect those which only exist inside the dipole. Additional experiments are planned to see if removing the coil ends will eliminate the axial oscillations and to investigate the effect of a heater on the portion of the coil inside the dipole. The very slow decay of these induced currents remains somewhat of a mystery since the contact resistance between strands is much too large to explain the long time constants observed.

\section{ACKNOWLEDGMENTS}

The authors would like to thank the cryogenics group of the RHIC Project for their help in performing these measurements. Joe D'Ambra of the Superconducting R \& D section operated the magnet and assisted in all phases of the experiment.

\section{REFERENCES}

[1] H. Brück et al., "Observation of a periodic pattern in the persistentcurrent fields of the superconducting HERA dipole magnets," DESY Repon HERA 91-01, 1991.

[2] A.K. Ghosh, K.E. Robins and W.B. Sampson, "Axial variations in the magnetic field of superconducting dipoles," Superconductor-4, Plenuon Press, NY, pp.765, 1992.

[3] A.K. Ghoah, K.E. Robins and W.B. Sampson, "Axial variations in the magnetic field of auperconducting dipoles and quadrupoles," Proceedings of the 1993 Particle Accelerator Conference, Washington, DC, pp.2742, 1993.

[4] A.K. Ghosh, K.E. Robins and W.B. Sampson, The ramp rate dependence of the sextupole field in superconducting dipoles," IEEE Transactions on Magnetics, vol. 30, no. 4, pp.1718, 1994. 


\section{DISCLAIMER}

This report was prepared as an account of work sponsored by an agency of the United States Government. Neither the United States Government nor any agency thereof, nor any of their Governes makes any warranty, express or implied, or assumes any legal liability or responsibility for the accuracy, completeness, or usefulness of any information, apparatus, product, or process disclosed, or represents that its use would not infringe privately owned rights. Reference herein to any specific commercial product, process, or service by trade name, trademark, manufacturer, or otherwise does not necessarily constitute or imply its endorsement, recomor favoring by the United States Government or any agency thereof. The views

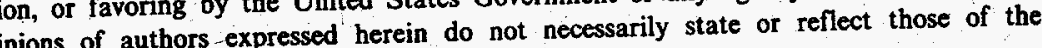
United States Government or any agency thereof. 


\section{DISCLAIMER}

Portions of this document may be illegible in electronic image products. Images are produced from the best available original document. 